\title{
Correction to: Prospective pediatric study comparing glomerular filtration rate estimates based on motion-robust dynamic contrast-enhanced magnetic resonance imaging and serum creatinine (eGFR) to ${ }^{99 \mathrm{~m}} \mathrm{Tc}$ DTPA
}

\author{
Sila Kurugol ${ }^{1}$ (D) - Onur Afacan ${ }^{1} \cdot$ Richard S. Lee $^{2} \cdot$ Catherine M. Seager $^{2} \cdot$ Michael A. Ferguson $^{3} \cdot$ Deborah R. Stein $^{3}$. \\ Reid C. Nichols ${ }^{4} \cdot$ Monet Dugan $^{1} \cdot$ Alto Stemmer ${ }^{4} \cdot$ Simon K. Warfield ${ }^{1} \cdot$ Jeanne S. Chow ${ }^{5}$
}

Published online: 13 March 2020

(C) Springer-Verlag GmbH Germany, part of Springer Nature 2020

Correction to: Pediatric Radiology (2020)

https://doi.org/10.1007/s00247-020-04617-0

The originally published version of this article contained a typographical error. In the text under the subheading "Dynamic contrast-enhanced MRI method, post-processing, and MRGFR calculation" and in Table 1 the intravenous injection rate of gadobutrol was incorrectly listed as $0.2 \mathrm{~mL} / \mathrm{s}$. The actual intravenous injection rate of gadobutrol was $2 \mathrm{~mL} / \mathrm{s}$.

Publisher's note Springer Nature remains neutral with regard to jurisdictional claims in published maps and institutional affiliations.

The online version of the original article can be found at https://doi.org/ $10.1007 / \mathrm{s} 00247-020-04617-0$

Sila Kurugol

sila.kurugol@childrens.harvard.edu

1 Computational Radiology Laboratory, Department of Radiology, Boston Children's Hospital and Harvard Medical School, 300 Longwood Ave., Boston, MA 02115, USA

2 Department of Urology,

Boston Children's Hospital and Harvard Medical School, Boston, MA, USA

3 Division of Nephrology,

Boston Children's Hospital and Harvard Medical School,

Boston, MA, USA

4 Siemens Healthcare, Erlangen, Germany

5 Department of Radiology,

Boston Children's Hospital and Harvard Medical School,

Boston, MA, USA 
Table 1 Imaging parameters

$\begin{array}{ll}\text { Sequence name } & \text { Dynamic radial VIBE stack-of-stars } \\ & \text { 3-D FLASH prototype sequence }\end{array}$

Total number of spokes (radial lines) acquired

3,978

Duration

$6 \mathrm{~min}$

Number of radial lines per volume

34

Temporal resolution

$\sim 3 \mathrm{~s} /$ volume

Voxel size

$1.25 \times 1.25 \times 3 \mathrm{~mm}$

Number of slices

32

Golden angle radial ordering

Yes

Repetition time (TR)

$3.56 \mathrm{~ms}$

Echo time (TE)

$1.39 \mathrm{~ms}$

Flip angle (FA)

$12^{\circ}$

Orientation

Offline image reconstruction algorithm

Contract injection time

Contrast dose

Coronal

Compressed-sensing reconstruction using regularization, i.e. total variation in temporal dimension [17] (using the MATLAB code provided by New York University that was modified for this purpose)

Contrast injected $20 \mathrm{~s}$ after the acquisition starts using a power injector

$1 \mathrm{~mL} / \mathrm{kg}$

$2 \mathrm{~mL} / \mathrm{s}$

Injection rate

3-D FLASH 3-dimensional fast low-angle shot, min minute, VIBE volumetric interpolated breath-hold examination 